\title{
ESTUDIO DEL BARRENADOR DE Alnus acuminata H.B.K. ("ALISO")*
}

\author{
Baltazar Castañeda, Hernán ${ }^{1}$
}

Facultad de Ciencias Forestales y del Ambiente

\begin{abstract}
RESUMEN
Se estudiaron aspectos de la biología y tipos de daños del escarabajo barrenador (Coleóptera: Cerambycidae), que está atacando al Alnus acuminata H.B.K. ("Aliso"), en plantaciones naturales situado en el distrito de Quichuay (Concepción), e Ingenio (Huancayo). Se muestrearon árboles al azar, según Sarmiento (1990); se capturaron individuos de diferentes estados utilizando la metodología de Fiorentino, et al (2004). Se observó la presencia de adultos, larvas y pupas; no hubo huevos. Los árboles atacados muestran una marchitez progresiva terminal, en la corteza presentan perforaciones de entrada y salida del escarabajo; si el daño es severo, hay desprendimiento agrietamiento con deformación de la superficie externa. Por debajo de la corteza, se observaron en la parte del tejido cambial larvas de varios tamaños barrenando la albura; y adultos de varios tamaños. En la parte basal del árbol, se observó indicios de aserrín fino como signo de la presencia del insecto. Hubo mayor intensidad de daño y presencia de individuos en los períodos de mayor humedad.
\end{abstract}

Palabras clave: barrenador, Alnus acuminata H.B.K, Cerambycidae, "Aliso".

\section{STUDY OF BORER OF Alnus acuminata H.B.K. ("ALDER")}

\begin{abstract}
Inportant aspects of the biology and types of damages that the beetle borer (Coleóptera: Cerambycidae), that is attacking the Alnus acuminata H.B.K. ("Alder"), in the natural plantation located in the district of Quichuay (Concepcion), and Ingenio (Huancayo). Some trees were tatting at random as sample, according to Sarmiento (1990); they captured individuals at different stages utilizing Fiorentino (2004). They observed the presence of adults, larva and pupa; there weren't eggs. The damages trees show a progressive withering terminal, they show perforations it the entry and coring of the beetle if the damage is severe these is a cracking are deformation of the external surface. Underneath the bark, they observed in the cambial tissue larvae of different size drilling the laburnum(sapwood); and adults of several sizes. In the basal part of the tree some residencies of fine sawdust which indications the presence of the insect there werw more severe damage and presence of these individuals insects in the principal period of humidity.
\end{abstract}

Key words: Beetle borer, Alnus acuminata H.B.K, Cerambycidae, Alder.

\footnotetext{
* Este trabajo de investigacion fue recibido el 20/12/2007, retornado para su revisión el 22/09/2008 y aprobado para su publicación el 27/02/2009.

1. E-mail: hbaltaza@gmail.com
} 


\section{INTRODUCCIÓN}

Las plantaciones naturales de Alnus acuminata H.B.K. ("Aliso"), situado en el Valle del Mantaro, están atacados por un escarabajo barrenador de la familia Cerambycidae (Coleóptera) Arizapana (1982); ocasionando daños a nivel del tejido cambial y albura. El daño por el insecto, sitúa en riesgo el crecimiento, producción maderable, rol ecológico y medicinal a los árboles afectados; además presentan una marchitez terminal a generalizada, y la especie se encuentra en peligro de extinción. Manta (2006) y Baltazar (2007), reportaron la presencia del insecto barrenador cerambícido en el estado larval en Quichuay (Concepción), Ingenio y San Pedro de Saño (Huancayo). Actualmente no se conoce la magnitud del impacto del daño por el insecto, su importancia económica y aspectos de su identificación y biología del insecto longicornio.

La investigación, tuvo como objetivo identificar a nivel de familia, describir los signos y síntomas que ocasionaban a las plantas de "aliso" afectado, y conocer algunos aspectos de la biología del insecto, considerando que el presente estudio servirá como un aporte para en el futuro desarrollar un programa de manejo integrado de plagas forestales en el Valle del Mantaro.

\section{MATERIAL Y MÉTODOS}

\section{Lugar de ejecución:}

La investigación se realizó en fase de campo en árboles de Alnus acuminata H.B.K. ("Aliso"), de plantaciones naturales situado en la margen del río Ingenio, distritos de Ingenio (Huancayo), y Quichuay (Concepción); y en el Laboratorio de Manejo Forestal de la Universidad Nacional del Centro del Perú.

\section{Materiales y equipos}

- Equipos: Estereomicroscopio Leitz de 180x, microscopio óptico de luz de 1000x, cámara fotográfica digital SONY de 8,2 megapixeles, y lupa de 10x.

- Instrumental: Pinza de punta fina, estilete, bisturí, tijera, aguja entomológica.

- Placas petri, porta y cubre objetivos, frasco letal, ocular micrométrico.
- Envases de cartón, etiquetas, plumón indeleble, formato de evaluación, y libreta de campo.

- Reactivos: alcohol $96^{\circ}$, agua destilada.

\section{Diseño metodológico}

Se empleó el método exploratorio; cuya población, estuvo conformada por árboles de "aliso", situado en Ingenio, Saños Grande (Huancayo); Huánchar, Matahuasi, Quichuay (Concepción), y las otras situadas en el ámbito del Valle del Mantaro, aquellos que presentaron signos y síntomas de la presencia del barrenador.

\section{Técnicas y procedimientos de recolección de datos}

Se muestreo al azar árboles atacados por el insecto barrenador. Se obtuvieron muestras biológicas de estado larva, pupa y adulto, siguiendo la metodología de Sarmiento (1990) y Fiorentino (2004), y muestras de diferentes estados biológicos colectados.

Se prepararon montajes, según Gaviño, (1977). Para los signos y síntomas, se empleó la metodología de Arguedas, (2006).

Las muestras de larvas, pupas y adultos colectados, se enviaron al Servicio Nacional de Sanidad Agraria (SENASA), para la identificación.

Además, se extrajeron las partes afectadas de la base del fuste, en donde se observó los orificios de entrada y salida del insecto barrenador; luego se realizó la descripción del tipo de daño causado.

\section{Se evaluó:}

- Identificación de la familia del insecto.

- Signos y síntomas de daños, causado por el escarabajo barrenador al árbol afectado.

- Biología del barrenador, características morfológicas externas en sus diferentes estados presentes.

\section{RESULTADOS}

\section{Identificación del insecto}

No se ha logrado la identificación a nivel de género y especie, del insecto longicornio, pese ha haber enviado muestras de individuos capturados al Laboratorio SENASA. 
De las muestras enviadas al SENASA (2007), se obtuvo la clasificación taxonómica:

\author{
Jerarquía taxonómica \\ Reino: Animalia \\ Phyllum: Arthropoda \\ Subphyllum: Hexapoda \\ Clase: Insecta \\ Subclase: Pterygota \\ Infraclase: Neoptera \\ Orden: Coleoptera \\ Suborden: Adephaga \\ Superfamilia: Chrysomeloidea \\ Familia: Cerambycidae \\ Genero: aun no reportado \\ Especie: aun no reportado
}

Chemsak, Linsley \& Noguera (1992); ITIS REPORT, (2007).

\section{Signos y síntomas de daños, causado por el escarabajo barrenador.}

Se han evaluado a plantaciones de aliso situadas en los distritos de Quichuay e Ingenio (cerca de la central hidroeléctrica), donde el $100 \%$ de los árboles de Alnus acuminata "aliso", esta siendo atacado por el barrenador; dichos arboles presentan una marchitez generalizada que avanza de la parte basal del fuste hacia la parte superior del árbol (ápice), muestran síntomas de decaimiento, rasgos de amarillamiento e incremento de la defoliación.

Se observa la presencia de perforaciones mayores a $3 \mathrm{~mm}$., que son orificios de entrada y salida del escarabajo longicornio. La abertura de entrada tiene forma oval elíptica a irregular, y esta situada en la corteza con prolongación hasta la albura del árbol.

Si el daño es severo se observa el desprendimiento de la corteza. Las perforaciones y grabaciones observadas al desprender la corteza, sobre el duramen expresa una línea zigzagueante a ondulada con profundidad mayor a 2,0 $\mathrm{mm}$ con presencia de abundante aserrín fino; esta característica puede observarse en la longitud del fuste del árbol afectado.

Se ha observado, una mayor severidad del daño en los meses de enero a marzo, y con mayor presencia de humedad ambiental, siendo la humedad un factor importante para la vida del insecto. En los meses de mayor frío y clima seco la población de barrenadores, disminuyó significativamente.
Los árboles atacados, presentaron indicios de aserrín fino color marrón claro a oscuro, por la presencia del daño por las larvas (Figura 7).

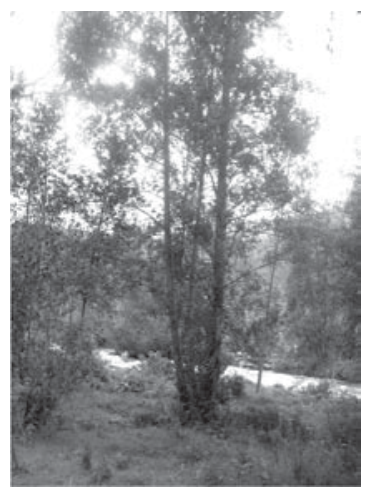

Figura 1. Árbol de Alnus acuminata H.B.K. ("Aliso"), con síntomas de amarillamiento $y$ marchitez terminal.

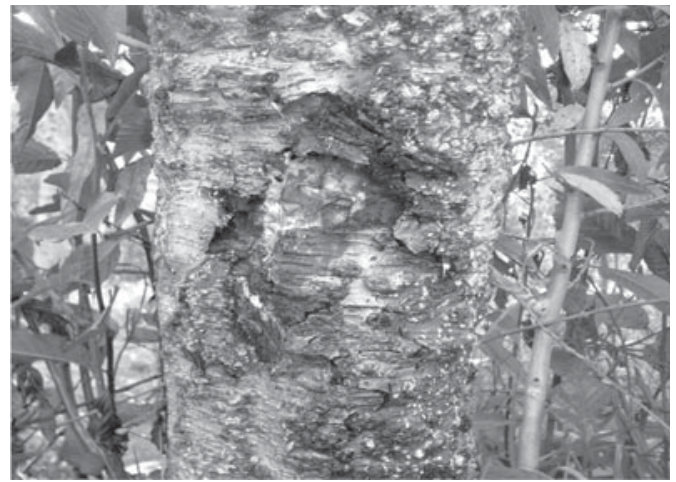

Figura 2. Daños a la corteza, se observa las grietas y deformación de la superficie externa.

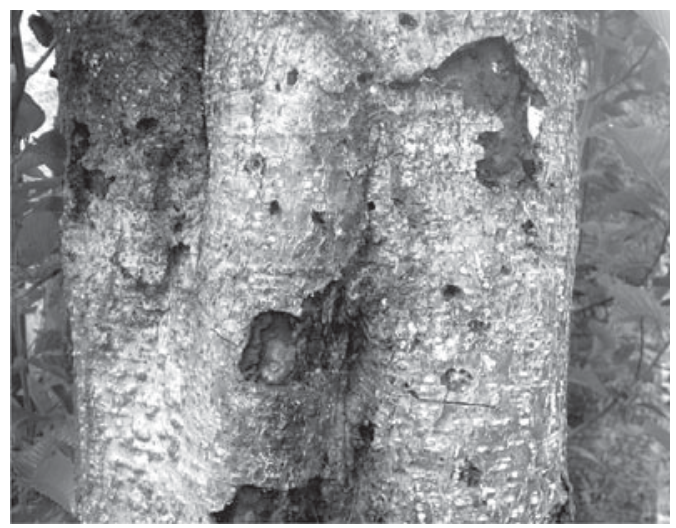

Figura 3. Orificio de entrada y salida del insecto adulto. 


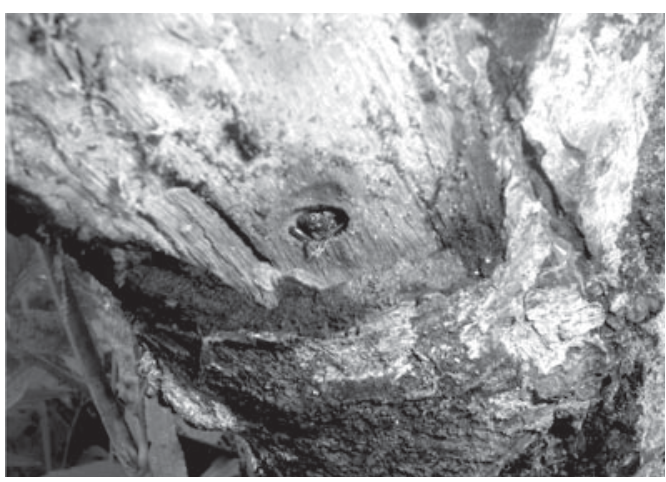

Figura 4. Adulto del barrenador en su galería.

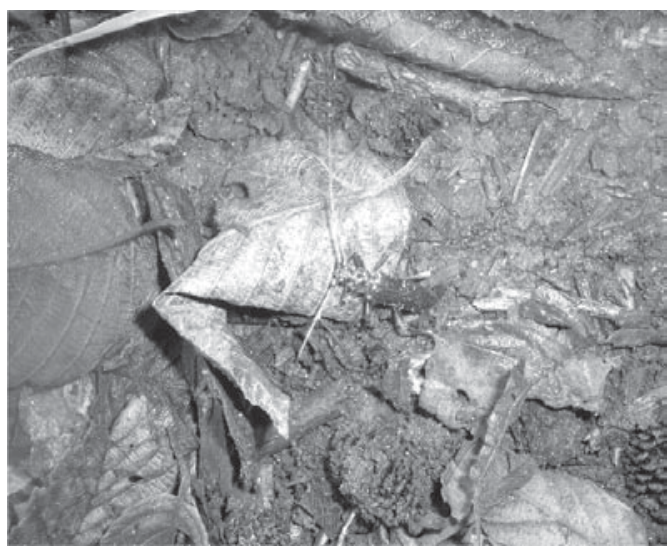

Figura 5. Adulto recién salido de la galería.

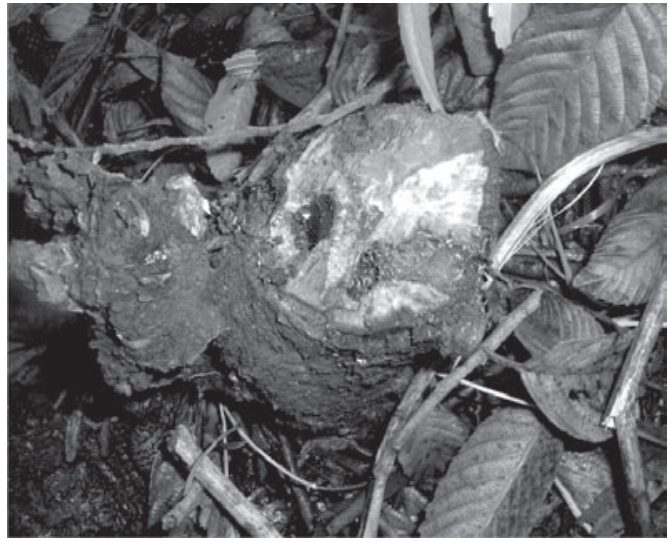

Figura 6. Daños de la larva, al tejido del cambium en el fuste del aliso, en la parte basal.

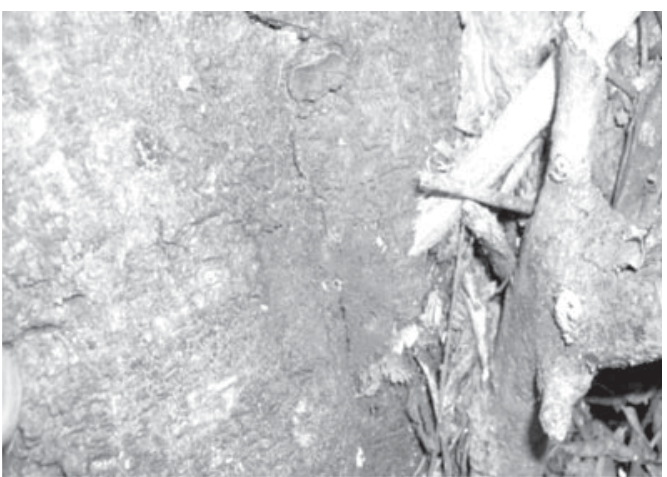

Figura 7. Signos de la presencia del barrenador en árbol afectado.

\section{Biología del barrenador, características morfológicas} externas

\section{Insecto adulto}

La forma del cuerpo del insecto es alargada, cuya longitud varia de desde 1,7 mm hasta mas de 2,7 mm; la cabeza por lado dorsal tiene una longitud de 1,7 mm. El ancho del cuerpo del insecto puede variar desde 2 $\mathrm{mm}$ en el lado de la cabeza hasta 5,00 $\mathrm{mm}$ en el lado del abdomen.

Presenta un par de antenas filiformes con 12 artejos, que sobrepasa la longitud del cuerpo del insecto; se observa que el segundo artejo es lobulado, y mide más de $15 \mathrm{~mm}$.

Presenta en el mesotórax dos alas élitro, y en el metatórax dos alas membranosas; los élitros presentan abundante pilosidad de color castaño claro, y presenta una estriación alargada de color marrón oscuro, una mancha alargada algo negruzca casi en el medio en una línea longitudinal.

En la unión del tórax y el abdomen un área característica cuya forma varía de cono a triangular de color amarillo. No presenta pigidium.

Color: el insecto presenta un color marrón claro a oscuro, con tonalidad cambiante hacia el lado de la cabeza que termina en ligeramente más oscuro visto del lado dorsal. Las patas y antenas son de color marrón claro (Figura 8). 


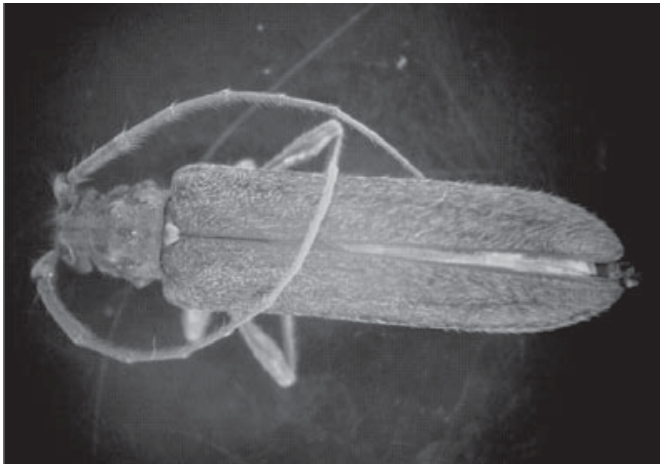

Figura 8. Estado adulto.

\section{Estado larva}

Las larvas observadas presentan tamaño variado de 2,0 a 3,0 mm, pudiendo alcanzar hasta $40 \mathrm{~mm}$; en algunos casos las larvas pequeñas adoptaron un color rosado rojizo, como respuesta a presencia de tanino en el proceso de la alimentación. Además, presentan una formación de mandíbulas grandes y fuertes, preparados para barrenar y formar galerías grandes. El lado de la cabeza, de la larva se encuentra ensanchado, característico de la familia de los cerambícidos.

La larva es vermiforme ensanchada en el lado de la cabeza; presenta formación de mandíbulas fuertes de color marrón claro, son minadoras de madera, gran desarrollo torácico y patas reducidas, reemplazadas por mamelones que le permiten reptar en las galerías donde viven, característico de los cerambícidos; cuyo tamaño puede variar del 2,00 mm hasta mas de $35 \mathrm{~mm}$, cuya coloración en general es amarillo blanquecino, con formación pronunciada de los segmentos abdominales y no tiene capacidad de locomoción (Figura 9).

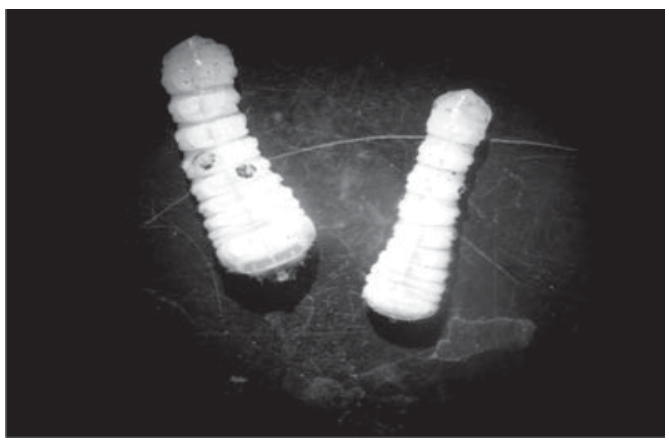

Figura 9. Estado larva.

\section{Estado pupa}

La pupa es de formación exarata, cuyo tamaño varía de 1,70 mm hasta más de 2,00 mm; cuya consistencia es suave. Son de color amarrillo claro y translucidos, y no presenta pilosidad. Se observó de varios tamaños, en donde algunos mostraron la formación de las antenas, setas en todo el cuerpo. La mandíbula observada es característica propia de la familia Cerambycidae (Figura 10).

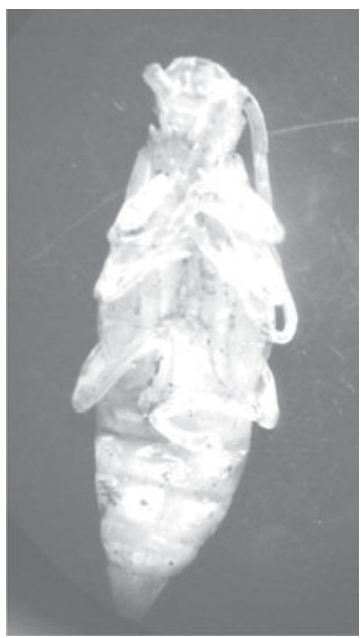

Figura 10. Estado pupa.

\section{DISCUSIÓN}

Los coleópteros son insectos de importancia forestal para la especie de Alnus acuminata H.B.K, como afirman Arguedas (2006), y Espinoza y Arguedas, (2005). Los Cerambycidae forman una familia muy numerosa $y$ muy variada. Todos sus miembros son fitófagos. La mayoría tienen antenas largas y el cuerpo de forma cilíndrica, alargada. La coloración es muy variada pero muchas especies presentan colores bonitos. Los adultos se encuentran sobre flores, ramas, troncos, follaje, bajo corteza; muchos son nocturnos. Las larvas son cilíndricas, siendo barrenadores de madera, cuya característica observada es similar a la reportada por Duffy (1957, 1960, 1963); para P. recurva y P. semipunctata. Algunas especies son de importancia forestal o agronómica. La organización de esta familia sigue el orden de Chemsak, Linsley y Noguera (1992); coincide en la jerarquía taxonómica con ITIS REPORT (2007). 
Las características morfológicas observadas, de pupa, larva y adulto coinciden ampliamente, con la observada por FAO (2007), y Dourojeanii, (1966), para la familia cerambicidae. Las larvas son de color blanco amarillento, con el extremo de la cabeza de color marrón.

Se observaron larvas que presentaban en el cuerpo manchas color rojo purpura a rosado, siendo probable la acumulación del semioquímico de tanino obteniendo un color fisiológico por alimentación.

La pupa es de color blanco cremoso, similar en forma y tamaño al adulto, y posee una consistencia gelatinosa, con una cubierta transparente y frágil. El cuerpo del adulto es de color marrón claro a oscuro, y presenta una mancha gris situada casi en la parte central de los élitros. No se observaron diferencia entre machos y hembras; y los insectos no soportaron crianza en cautiverio.

Con relación al hábito alimenticio, las larvas mostraron preferencia alimentaria, al tejido del parénquima, este es similar a la reportada por Dourojeanii, (1966), para la especie de Phoracanta semipunctata (Coleóptera: cerambycidae), en Perú que es el escarabajo taladrador del Eucalyptus globulus Labill.

Los árboles afectados, presentan una marchitez generalizada, esto debido a que las larvas y el adulto se alimentan barrenando el tejido del parénquima, y que puede llegar hasta el xilema o floema, resultando que el árbol presentara deficiencias de nutrientes a través del sistema vascular, como afirman Dourojeanii, (1966) y Espinoza y Arguedas, (2005).

La presencia de aserrín fino como signo, de la presencia del insecto, es característico y similar a la observada por Arguedas (2006), y Espinoza y Arguedas, (2005), para el Alnus acuminata en Costa Rica; y fueron propios de los escarabajo taladradores y barrenadores como el Scolytodes alni (Coleóptera. Scolytidae).

Además, se ha observado, mayor severidad y niveles de daños e individuos de larva, pupa y adulto, en época de mayor humedad y precipitación, esto coincidiendo con la reportada por Arguedas (2006), y Espinoza y Arguedas, (2005), para el Alnus acuminata.

\section{CONCLUSIONES}

- El insecto barrenador del Alnus acuminata H.B.K. ("Aliso"), pertenece a la familia Cerambycidae y Orden Coleóptera, es considerado una especie nueva no identificada reportada para el Perú.

- Los árboles afectados por el escarabajo barrenador, ha situado a la especie en peligro de extinción, en razón a que el $100 \%$ de las plantaciones localizadas en Quichuay e Ingenio, presentaron signos y síntomas por el daño del insecto.

- La marchitez generalizada y defoliación, que presentan los árboles atacados, se debe a que las larvas se alimentan del tejido del parénquima, alterando su nutrición por el sistema vascular de la planta.

- Las perforaciones y grabaciones observadas al desprender la corteza, sobre la albura expresa una línea zigzagueante a ondulada con profundidad mayor a dos milímetros, con presencia de abundante aserrín fino; esta característica puede observarse en la longitud del fuste del árbol afectado, por debajo de la corteza.

- Se ha observado, con mayor agresividad los niveles de daño en los meses mayor lluvia y humedad relativa, siendo la humedad un factor importante para la vida del insecto

\section{LITERATURA CITADA}

Arguedas, M. 2006. Clasificación de tipos de daños producidos por insectos forestales. Primera Parte Solución Tecnológica. Kurú: Revista Forestal (Costa Rica) 3(8).

Arguedas, M. 2006. Clasificación de tipos de daños producidos por insectos forestales. Solución Tecnológica. Segunda Parte. Kurú: Revista Forestal (Costa Rica) 3(9).

Baltazar, H. 2007. Entomología general y forestal. compendio. Facultad de Ciencias Forestales y del Ambiente. UNCP. 145 pp. 
CFI-Technical Pest Research. 2007. Plagas de la madera (en línea). Disponible en: http//: www. Cfiplagas.com.ar/madera.htm. Visitado: 14-12-07.

Chemsak, Linsley \& Noguera. 1992. Listados faunísticos de México. II. Los Cerambycidae y Disteniidae de Norteamérica, Centroamérica y las Indias Occidentales (Coleóptera). Instituto de Biología, UNAM, México, 204 p.

Crespo,J.y Martínez, J. 2007. Escarabajos longicornios. Fauna lbérica.

Dourojeanii, M. 1966. El taladro de los eucaliptos Phoracantha semipunctata (Fabricius) (Coleóptera: Cerambycidae) Nuevo Registro en el Perú. Revista Forestal del Perú. V. 1: 1-12.

Duffy, E., 1957. A monograph of the immature stages of African timber beetles (Cerambycidae). British Museum, London, pp. 38-41; 113-117.

Duffy, E., 1960. A monograph of the immature stages of neotropical timber beetles (Cerambycidae). British Museum, London, 327p.

Duffy, E., 1963. A monograph of the immature stages of Australasian timber beetles (Cerambycidae). British Museum, London, pp. 68-74.

Espinoza, A. y Arguedas, M. 2005. Evaluación de factores que favorecen el ataque Scolytodes alni (Curculionidae, Scolytinae) en plantaciones de jaúl (Alnus acuminata Kunth) en Costa Rica. Nota Técnica. Kurú: Revista Forestal (Costa Rica) 2(5).
FAO. 2007. Fores pest species profile. [on line]. Phoracantha recurva Newman, 1840 and Phoracantha semipunctata (Fabricius, 1775). November. url: [www.fao.org]. Visit: [21-102007].

Fiorentino, D. B. y Diodato, L. 2004. Métodos de recolección de Torneutes pallidipennis Reich 1837. (Coleoptera:Cerambycidae) en Santiago del Estero. Quebracho 11: 54-59. Argentina.

FORESTAL Y FAUNA. 1982. Semana Forestal Nacional. Boletín extraordinario. El aliso y la guinda en proceso de exterminio. Ministerio de Agricultura. Huancayo. Perú. 13 p.

ITIS REPORT. 2007. [Documento en Linea]. NODC Taxonomic Code, database (version 8,0). Acquired: 1996. Disponible en < http://www. itis.usda.gov/index.html>. [Consulta: 15-03-07].

Manta, M. 2006. Comunicación personal. Visita Técnica al Valle del Mantaro. Setiembre 2006. Curso Protección Forestal. Universidad Nacional Agraria La Molina. Lima. Perú.

Sarmiento, M. J. 1990. Evaluación de plagas. Copia mimeografiada del Curso de Evaluación y Crianza de Insectos. Universidad Nacional Agraria La Molina. Lima. Perú. 9 p. 